\title{
Etiology of Multiple Sclerosis
}

Lundsgaard $\mathrm{E}^{*}$

Tolvkarlevej, Hilleroed, Denmark

*Corresponding author: Lundsgaard E, Tolvkarlevej, Hilleroed, Denmark, Tel: 2169705240; E-mail: ebbe-lone@tanke.dk

Received date: October 06, 2017; Accepted date: November 09, 2017; Published date: November 16, 2017

Copyright: (C) 2017 Lundsgaard E. This is an open-access article distributed under the terms of the Creative Commons Attribution License, which permits unrestricted use, distribution, and reproduction in any medium, provided the original author and source are credited.

\begin{abstract}
Multiple Sclerosis (MS) is characterized by degradation of the essential myelin sheath around the nerves in the white matter of the central nervous system (CNS). I argue that this is caused by insufficient production of myelin, as myelin-forming oligodendrocytes become damaged by excess steroid receptors type 1 in the cytoplasm, which clump and release zinc due to the relative lack of their ligands. This may gradually lead to hyperphosphorylation of tau protein. Accordingly, the etiological basis for MS is thought to be at least three synergistic systems with the components, steroid receptors type 1 and their ligands: vitamin D, estrogen and testosterone. To prove this hypothesis with its many variables, it has been necessary to use a system of nonhomogeneous differential equations.
\end{abstract}

Keywords: Alzheimer's disease; Amyotrophic lateral sclerosis; Vitamin D; Sex hormones

\section{Introduction}

This novel hypothesis is based on established data described in the literature, data that make up the pieces for solving the MS puzzle with the oligodendrocyte as object. It is diametrically opposed to the current MS concept, which hypothesizes that the degradation of myelin is due to an immunological response.

Because my hypothesis is unique and lacks references in the literature, it is opportune to integrate my research of Alzheimer's Disease (AD) [1] and Amyotrophic Lateral Sclerosis [2] (ALS). These diseases share determinants with MS, characterized by an increased ratio of steroid receptors type 1 to sex hormones in the neurons of CNS. This also happens in the oligodendrocytes, which further are specifically vulnerable due to the content of vitamin D receptors. However, although MS's pathology in the fertile age is thought to depend mainly on the relationship between vitamin $\mathrm{D}$ and its receptors, it recalls $\mathrm{AD}$, especially in older men and postmenopausal women. This raises the question of involvement of neurons belonging to the gray matter.

It is known that oligodendrocytes, other glial cells, and neurons in the CNS have a high metabolic rate and, with certain exceptions, are equipped with a large number of steroid receptors type 1, which are freely movable in cytoplasm.

ALS constitutes such an exception and occupies a key position as there is a very high concentration of receptors for testosterone/ dihydrotestosterone in the diseased nerves, whereas ALS does not occur in nerves free of androgenic receptors [3] for example in the nerves responsible for the movements of the eyes.

With regard to $\mathrm{AD}$ and its predilections it is likely that the steroid receptors in hippocampus predominantly are estrogenic, but rather androgenic in lobus frontalis. Moreover, the formation of extracellular plaques occurs notably in AD. Regarding MS, the interaction with both estrogen and androgens discussed below in the section of 'Sex hormones' confirms that both types of receptors, besides vitamin D receptors, are represented in the oligodendrocytes.

All steroid receptors type 1 bind to specific steroids that enters the cell from the blood. The resulting complex is subsequently translocated to DNA in the cell nucleus, whereas unliganded steroid receptors with or without heat shock protein might form lumps [4] in the cytoplasm.

However, the deciding pathological factor in MS, AD and ALS appears to be the release of zinc from the lumps or receptor remnants, as each steroid receptor is provided with four zinc atoms [5]. Zinc has been shown to damage the microfilaments inside the oligodendrocytes [6] as well as those inside the neurons [7] in AD and ALS.

Other determinants comprise gender distribution and reproductive age:

Women are overrepresented in MS and $\mathrm{AD}$, whilst men dominate in terms of ALS.

$\mathrm{AD}$ and ALS are thought to arise when the production of sex hormones decrease with age in persons genetically predisposed for a high ratio of receptors to hormones.

The preponderance of MS in the fertile age is explained in the next section.

\section{Vitamin D}

Sunlight is the most potent source for the synthesis of vitamin D steroid, and the farther away you live from equator, the higher the risk of MS. A study of seven million military personnel [8] in the United States revealed that the vitamin D content in the blood is inversely proportional to the occurrence of MS, which in women is approximately three times as frequent as in men.

MS occurs predominantly in the fertile age [9] and the enhancement [10] of vitamin D receptors caused by estrogen is probably pathognomonic. Indeed, vitamin D receptors are significantly more widespread (Results) [11] in MS lesions-particularly new, active lesions-compared to healthy brain tissue in humans. 


\section{Sex hormones}

The production of free estrogen in women decreases by a factor of about twelve after menopause, whereas men's production of free estrogen only decreases slightly and, moreover, only accounts for about one fourth of the level of the fertile woman.

Pregnant women with MS might exhibit an improvement of the symptoms late in pregnancy, where the production of estrogen is very high. The reason is assumed to be the inverse relationship [12] between hormones and receptors, as well as the high receptor binding capacity (Discussion, end) [13] in women, both of which reducing the number of unbound estrogen receptors. Presumably, this takes precedence over a marginally further increase of vitamin D receptors in fulminant MS.

Women with MS also have a lower testosterone concentration [14] than normal, which, according to the inverse relationship between hormones and receptors causes an increase in the androgenic receptors, thereby creating a cumulative effect in conjunction with the vitamin D receptors.

Men with MS and high level of estrogen [15] may have AD-like symptoms, while testosterone [16] supplementation over some time shows cognitive improvement and decreasing brain tissue loss. This indicates that the amount of unbound androgenic receptors is reduced, which also attenuates the effect of the estrogen-induced vitamin D receptors.

\section{Proof}

In $\mathrm{AD}$ and $\mathrm{ALS}$, the mathematical model is derived from the probability distribution of sex hormones in a population. The sex hormone receptors are distributed in the same way, just as a reflection of the sex hormones, why the proportion between hormones and receptors in a subpopulation allows the estimation of the applied rate constants.

The distribution of vitamin $\mathrm{D}$ receptors in the population is not defined, but the following system of nonhomogeneous differential equations circumvents this using the bare ratio of vitamin $\mathrm{D}$ receptors (x) to vitamin $\mathrm{D}(\mathrm{y})$, shown by the fraction $\mathrm{x} / \mathrm{y}$.

In the first and third equations it is assumed that $\mathrm{y}$ is in deficit, whereby all $\mathrm{y}$ bind to an equivalent fraction of $\mathrm{x}$, which gives $a \mathrm{x} \bullet \mathrm{y}$ or $y \bullet y$ because $a x=y$. The minus sign $-y \bullet y$ or $-y^{2}$ expresses that the link moves from the cytoplasm into the cell nucleus without participating in MS. The term $(x-a x)=(x-y)$ is the number of remaining $x$.

\section{Hence:}

In the second equation it is assumed that $\mathrm{x}$ is in deficit compared to $2 \mathrm{y}$, whereby all receptors $(\mathrm{x})$ bind to an equivalent fraction of $2 \mathrm{y}$ giving $2 \beta y \cdot x$ or $x \cdot x$ because $2 \beta y=x$.

As above, $-\mathrm{x} \cdot \mathrm{x}$ or $-\mathrm{x}^{2}$ is translocated into the cell nucleus. The term $(2 y-2 \beta y)=(2 y-x)$ is the number of remaining $2 y$. Hence:

The amount of estrogen is expressed at $\mathrm{z}$ and the frequency of MS is expressed by the independent variable, $s$.

The first equation shows a fertile woman with a normal level of estrogen (8z), but deficient in vitamin D (y). The disease rate is 3 s, i.e., 3 times men's.

The second equation shows a postmenopausal woman without MS and with normal levels of vitamin D (2y) and sex hormones (z).
The third equation shows a man with normal level of estrogen (2z), but deficient in vitamin $\mathrm{D}(\mathrm{y})$. The disease rate is s.

The method is legitimate, even though it is presumed that either y or $\mathrm{x}$ will be exhausted completely. In fact, only a fraction of both interacts with each other, as is the case with the probability distribution in $\mathrm{AD}$ and ALS. Adding appropriate rate constants to $\mathrm{y}$ and $\mathrm{x}$ elongated the curves, but did not change their tendency (Figure 1).

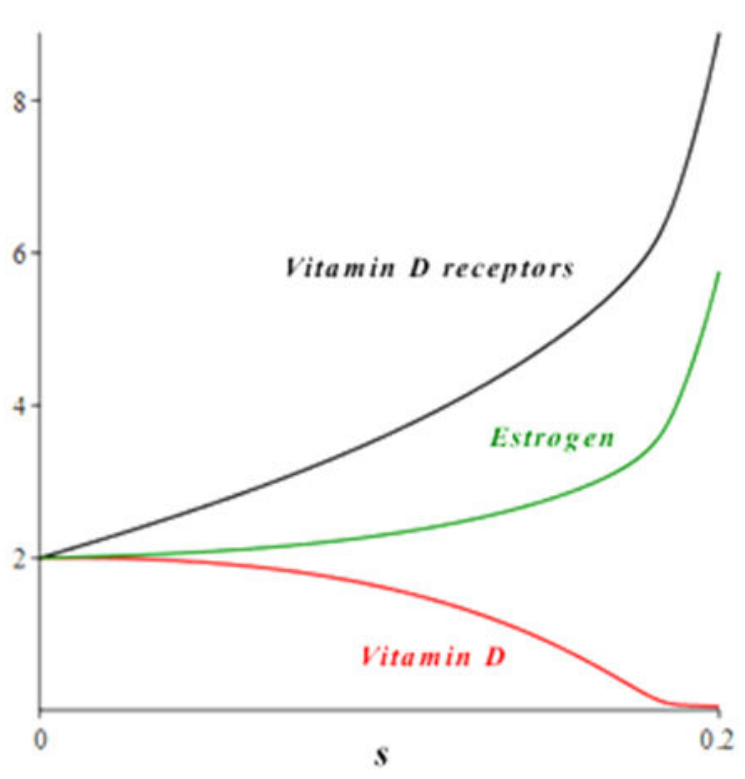

Figure 1: The abscissa, s, shows the increase of MS in accordance with the diverging curves based on the above equation system and solved by aid of MAPLE. The augmented rise of estrogen near 0.2 would cause a reduction of estrogen receptors that counteracts the pathological effect of vitamin $D$ receptors. It has been necessary to insert 'boundary values', which means that $\mathrm{x}, \mathrm{y}$ and $\mathrm{z}$ have the value 2 when $s$ has the value zero. However, the tendency of the curves is not changed.

\section{Discussion}

The increasing ratio of unbound vitamin $\mathrm{D}$ receptors to the level of vitamin $\mathrm{D}$ is convincingly correlated with the risk of MS, and this ratio grows faster by free estrogen in the blood.

Such an excess of receptors can aggregate and is able to release zinc, which demonstrably plays a role in the hyperphosphorylation of tau protein in neurons. This happens in the oligodendrocytes too, as a prelude to the progressive development of MS.

The relative increment of vitamin $\mathrm{D}$ receptors caused by estrogen is probably the most significant feature of MS in the fertile age. However, the overall pool of unbound steroid receptors plays an important role, especially at aging in persons hereditary inclined for low levels of sex hormones against high levels of receptors. This group can exhibit progression of MS resembling the pathology of $\mathrm{AD}$, why it is conceivable that neurons in specific areas of the brain get involved.

Certain therapeutic measures seem possible within the framework of this hypothesis. Among other things, some drugs prevent the clumping of steroid receptors upon binding to them. 
MS appears to be related to polygenes, i.e., several genes responsible for the variation in the amount of sex hormones and their receptors, which approximates a probability distribution in the population. Optionally, vitamin D receptors are also subject to this distribution, whereas vitamin $\mathrm{D}$ is determined by external factors.

MS is hardly an autoimmune disease, a theory that has emerged for decades on a regular basis. On the other hand, the internal changes of the oligodendrocytes and the subsequent myelin degradation might provoke an immunological response in the CNS.

\section{References}

1. Lundsgaard E (2016) Alzheimer's, caused by steroid receptors. Denmark.

2. Lundsgaard E (2016) ALS and sex hormones. Denmark.

3. Weiner LP (1980) Possible role of androgen receptors in amyotrophic lateral sclerosis. A hypothesis. Arch Neurol 37: 129-131.

4. Vector $A B$ (2008) LLC: Role of Hsp90 machinery in native folding and activity of steroid/nuclear receptors.

5. Biology Pages (2011) Steroid hormone receptors and their response elements.

6. Richter-Landsberg C (2016) Protein aggregate formation in oligodendrocytes: Tau and the cytoskeleton at the intersection of neuroprotection and neurodegeneration. Biol Chem 397: 185-194.
7. Björkdahl C, Sjögren MJ, Winblad B, Pei JJ (2005) Zinc induces neurofilament phosphorylation independent of p70 S6 kinase in N2a cells. NeuroReport 16: 591-595.

8. Munger KL, Levin LI, Hollis BW, Howard NS, Ascherio A (2006) Serum 25-hydroxyvitamin D levels and risk of multiple sclerosis. JAMA 296: 2832-2838.

9. Nocoletti A (2001) Prevalence and incidence of multiple sclerosis in Catania, Sicily. Neurol 56: 62-66.

10. Faye EN, Karen M (2009) Estrogen controls vitamin D3-mediated resistance to experimental autoimmune encephalomyelitis by controlling vitamin D3 metabolism and receptor expression. J Immunol 183: 3672-3681.

11. Multiple Sclerosis Society of Canada (2015) Early stage study shows that vitamin $\mathrm{D}$ can promote myelin repair.

12. Klinge CR, Glob C (2008) The steroid hormone receptors. Libr women's med.

13. Tomassini V, Onesti E, Mainero C, Giugni E, Paolillo A, et al. (2003) Sex hormones modulate brain damage in multiple sclerosis: MRI evidence. BMJ Journals. J Neurol Neurosurg Psychiatry 76: 272-275.

14. Miranda H (2005) Sex hormones may affect multiple sclerosis. Front Biosci 14: 4477-4515.

15. Multiple Sclerosis Trust (2015) Hormones.

16. Dal Bianco A, Bradl M, Frischer J, Kutzelnigg A, Jellinger K, et al. (2008) Multiple sclerosis and Alzheimer's disease. Ann Neurol 63: 174-183. 